\title{
Investigating Older Adults' Preferences for Functions within a Human-Machine Interface Designed for Fully Autonomous Vehicles*
}

\author{
Alexandra Voinescu ${ }^{1[0000-0001-7689-335 X]}$, Phillip L. Morgan ${ }^{20000-0002-5672-0758]}$, Chris Al- \\ ford ${ }^{1}$ and Praminda Caleb-Solly ${ }^{10000-0001-8821-0464]}$ \\ ${ }^{1}$ University of the West of England, Bristol BS16 1QY, UK \\ alexandra.voinesculuwe.ac.uk \\ chris.alfordeuwe.ac.uk \\ praminda.caleb-solly@uwe.ac.uk \\ ${ }^{2}$ Cardiff University, School of Psychology, Cardiff, CF10 3AT, UK \\ morganphilecardiff.ac.uk
}

\begin{abstract}
Compared to traditional cars, where the driver has most of their attention allocated on the road and on driving tasks, in fully autonomous vehicles it is likely that the user would not need to intervene with driving related functions meaning that there will be little need for HMIs to have features and functionality relating to these factors. However, there will be an opportunity for a range of other interactions with the user. As such, designers and researchers need to have an understanding of what is actually needed or expected and how to balance the type of functionality they make available. Also, in HMI design, the design principles need to be considered in relation to a range of user characteristics, such as age, and sensory, cognitive and physical ability and other impairments. In this study, we proposed an HMI specially designed for connected autonomous vehicles with a focus on older adults. We examined older adults' preferences of CAV HMI functions, and, the degree to which individual differences (e.g., personality, attitude towards computers, trust in technology, cognitive functioning) correlate with preferences for these functions. Thirty-one participants $(M$ age $=67.52, S D$ = 7.29), took part in the study. They had to interact with the HMI and rate its functions based on the importance and likelihood of using them. Results suggest that participants prefer adaptive HMIs, with journey planner capabilities. As expected, as it is a CAV HMI, the Information and Entertainment functions are also preferred. Individual differences have limited relationship with HMI preferences.
\end{abstract}

Keywords: Connected Autonomous Vehicles, Human Machine Interface, Older Adults.

\footnotetext{
* We are thankful to our collaborators at Designability UK (Jessica Ridgers, Keir Haines, Hazel Boyd) for designing the HMI and collaborators at Transport Systems Catapult UK (Alan Nettleton, Duncan Napier, and Sophie N'Guende) for programming.
} 


\section{Introduction}

\subsection{Autonomous Vehicles, Mobility and Older Adults}

Autonomous vehicles (AVs) and connected autonomous vehicles (CAVs) are a hot topic with research, and development in this area has been soaring over the past 10years or so [1,2]. In February 2018, a Google Scholar search for publications containing the keywords 'autonomous' and 'vehicle' returned 2,060,000 results including multiple journal articles, conference papers, and reports. CAVs promise a reduction in road vehicle incidents, accidents (injuries and fatalities), potential for improving traffic flow, as well as allowing users to engage in other activities (e.g., social, work, rest) whilst being driven in a CAV. In addition, they also open up the opportunity of mobility for more people including those who cannot drive and/or are contemplating giving up driving. One large and growing sector of the population within most developed countries who will likely benefit from CAVs are older adults.

According to World Health Organization (2015) by 2050 the world's population over 60 years will double itself. It is expected to total 2 billion compared to 900 million in 2015 with 125 million people aged 80 years or older. There is a crucial need to ensure a means of staying and/or becoming mobile for this population sector such that they can better and more easily enjoy mobility with likely positive effects on factors such as social inclusion (reducing social isolation), well-being, and the ability to continue to contribute to the economy [3-5].

One important promise for the rise of CAVs is the decrease in car crashes [6], as human error is held responsible for most incidents and accidents [7]. One of the most vulnerable populations are older drivers, and particularly individuals above 65 years of age [8]. Thus, from a safety perspective alone, it is highly likely that older adults will benefit enormously from AVs. Similarly, some older adults might have to give up their driving licence due to health-related issues [9]. Car ownership, mobility and the ability to drive are associated with quality of life, with many that give up driving feeling older than their chronological age [10]. Driving cessation is also factor that contributes to health problems and a decline in well-being, including depressive symptoms [4,11]. Furthermore, research suggests that older adults (past drivers and non-drivers) are much more vulnerable to cognitive decline [12] meaning that factors such as attention, inhibition, memory, judgement and decision making can impair the ability to drive effectively and safely. CAVs - particularly those that are highly automated (e.g., Society of Automotive Engineers (SAE) Level 4, 2016) or fully automated (e.g., SAE Level 5, 2016) [13] should help to circumvent many of these issues and improve older adults' mobility. However, the psychological, cognitive and sensory factors that result in cessation of driving, still need to be considered for CAV HMI design as these will still impact the ability to interact with an HMI, and also result in additional support and functionality.

The main aim of the current paper is to present findings on an aspect of a connected autonomous vehicle (CAV) human-machine interface (HMI) developed as a major component of an Innovate UK funded project (Flourish) for use by older adults within Level 4-5 CAVs. This was an evaluation study where users experienced simulated fully 
autonomous CAV journeys with a basic HMI (containing e.g., a dynamic map, vehicle status features, stop button, and so on) being developed throughout the Flourish project. Immediately after, they were presented with a number of other features and functions (designed for a future trial(s)) on an interactive screen and asked a number of questions regarding preferences and usability. We also examined the degree to which individual differences (e.g., personality, attitude towards computers, trust in technology, cognitive functioning) correlate with preferences for these functions. The HMI design was informed by a literature review [1], public engagement workshops conducted part of the Flourish project and subsequent synthesis of best practices for the design of CAV HMI for older adults. We focus here on the evaluation of a first-generation CAV HMI with three main categories of functions: My Profile, Information and Entertainment, and, Future Journey Planning. Each had a drill-down facility to explore related functions. My profile contained: About Me (e.g. home address, contacts, health profile) and Preferences (e.g. contrast, brightness, text size). Information and Entertainment had Television, Internet Search, News, Weather, and Music. Future Journey Planning contained Create Future Journey, Planned Journeys, and, Edit Journeys.

\subsection{Autonomous Vehicles and Human-Machine Interfaces}

Autonomous vehicles have different levels of automation ranging from 0-5 [13]. Levels 1 and 2 represent having an autonomous feature (L1) or features (L2) such as adaptive cruise control, self-parking, and lane keeping assistance. Level 3 vehicles can drive in autonomous mode some/most of the time, although there will be a fallback in that a human driver can and might need to retake vehicle controls (known as handover from autonomous to manual driving) at some point(s) during journeys [14]. In terms of Level 4 and 5 CAVs (the focus of the current research), the human user (i.e., older adults in this case) does not have to engage in driving/controlling (or intervening/handovers) the vehicle so that their 'eyes' and 'mind' can be off the road [13]. Users of a Level 4 CAV might still choose to engage with some functions (e.g., change speed, change route, request a stop, and so on) and ignore others, whereas users of a Level 5 CAV should (in principle) not need to engage with any aspect of the driving task (i.e., the CAV is in control and makes all decisions) although like Level 4, CAV users might wish to engage in other in-vehicle technology enabled activities that are now possible because they do not need to drive the vehicle (e.g., infotainment). Also, and under various circumstances (e.g. route and system requirements), the user might choose to prioritize some functions and ignore others (e.g. check for places to park whilst ignoring estimated time of arrival). Consequently, CAVs require specially designed HMIs or dashboards that respond to users' needs and preferences, as well as ensuring that they are kept updated with pertinent CAV related information which might impact on their experience and require their input. Therefore, designers, engineers and other researchers (including human factors experts) need to have an understanding of what is actually needed or expected by potential users and how to balance the type of functionality they make available. 
To date, research has focused more on driving performance (e.g., speed, response time to critical situations, braking) across the lower levels (L1-L3) of vehicle automation $[15,16,1,17]$ and on human factors related variables that are important in automation (e.g., situation awareness and mental workload) [18-20]. There has been less focus on principles relating to the design and adaptation of CAV HMIs for vehicles with higher or indeed fully (Level 5) automated capabilities, and less again on designing and testing these with particular end-user groups in mind $[2,1]$.

\subsection{The Importance of Considering Individual Differences when Designing CAV HMIs for Older Adults}

Aging is associated with a series of biological and psychological impairments [21,22]. Cognitive aging appears in both normal and pathological ageing, as a consequence of brain changes caused by ageing. Not all cognitive functions are affected in the same way resulting in differences in impairment across the population and indeed varying within people due to medication or fatigue [23,24,22,21,25]. Well documented changes in cognitive functions are those of attention, working memory, executive functioning and other memory processes [26,27]. Together with changes in cognitive functioning, changes in sensory functions also tend to be more prevalent as we age. For example, visual, auditory and olfactory perception, as well as mobility and balance are negatively influenced by ageing [28-32].

HMI developers and researchers have already acknowledged the need to consider cognitive and sensory changes that might affect, for example, usability [33-36]. A recent literature review conducted by Morgan et al. [1] synthetized the numerous design principles of relating to HMI design for older adults with a focus on those being relating to CAV HMI design. These principles can largely be grouped into four categories: usability, accessibility, functionality, and adaptability. Accessibility and usability requirements of older adults' include some of the following principles: use of simple displays, reduce screen clutter and distractors, use of familiar conceptual models and presenting information (including items) as clearly as possible, using touchscreens and enhanced voice interaction [35,1]. For functionality, HMIs developers should consider functions that enrich in-vehicle safety (e.g. night vision enhancement, collision warning, health monitoring) [37,38]. Requirements for adaptability mostly refer to the ability to select and personalize screens, settings and mode of interaction (see [1] for a full review). However, Morgan et al. [1] note that most of the HF principles being incorporated into the design of HMIs for AVs and CAVs are quite generic and not specifically focussed on particular sections of the population and rarely consider other individual differences, such as those relating to cognitive ability. There is a gap in the consideration of design of AV/CAV HMIs for older adults at an individual as well as population level. Similarly, Molnar \& Eby [39] recently concluded that most of the HMI principles and guidelines for older drivers do not address sensory, physical and cognitive impairments associated with older age.

Beside cognitive functioning, other personal characteristics might influence peoples' expectations and experience with AV/CAV HMIs, and again this is an under-researched area. Most studies focus on ergonomic factors [40] and general personal characteristics 
such as age, gender, and values [41]. Other individual factors such as personality, attitudes, and trust seem to be largely over-looked. Despite this, researchers such as Engström et al.[42] have suggested that personality traits such as risk taking might influence the acceptance of driver support functions. As such, there is likely to be a link between acceptance and preference of various HMI functions (e.g. health monitoring, emergency stop) and usability (e.g. preference for simple or more complex information) and personality traits. As an example, those who score high on sensation seeking (a risk taking associated personality trait) might prefer more complex HMIs as they usually desire complex stimuli $[43,44]$. Those who score high on anxiety or emotional instability might prefer straightforward functions and be more in control as they need to be reassured due to a tendency to worry excessively [45]. For example, they might prefer $\mathrm{AV} / \mathrm{CAV}$ HMIs with easy to access information and possibly functions (that can be explored) for important yet basic information, such as vehicle status and speed.

Also, attitudes towards computers and trust in technology might also shape users' AV/CAV HMI-related preferences and subsequent experience with HMIs implemented into AVs/CAVs. Trust in autonomous systems is another key variable that influences user experience $[46,47]$. Trust appears to predict the likelihood of use of automation [48] there is some support that favors a well-designed HMI as a mediator of trust in automation [46]. As an example, adding speech output to an HMI ('HMI talks') can increase trust in automation [49]. Thus, it is likely that a higher level of trust in technology and/or automation will be related to better user experience and higher usability ratings of functions. Finally, attitudes towards computers can shape users HMI preferences, as technology related variables predict the use of technology [50,51]. Although there is no support for the assumption by some that older people do not accept new technologies, they are more willing to use them if the interface suits their abilities and needs [52,53]. Also, prior contact and interaction with computers improves attitudes and user behaviour [54]. Therefore, it is highly likely that attitudes towards computers will be related to CAV HMI design preferences and user experience.

\section{Goals and Objectives}

The current study was conducted due to the lack of research-to-date on the design and testing of CAV HMIs designed with, and for, older adults. Our main objective was to examine older adults' preferences and evaluation of CAV HMI features and functions, and analyse, the degree to which individual differences (e.g., personality, attitude towards computers, trust in technology, cognitive functioning) correlate with preferences for these functions.

\section{$2 \quad$ Method}

\subsection{Participants}

Thirty-one participants, mostly older adults, aged between 47 and 83 years old $(M=$ $67.52, S D=7.29$ ), took part in the study. This sample size is large enough to detect large effect sizes $(r=.50)$ with power of .8 [55]. The majority were male $(\mathrm{N}=19$, 
61.3\%). Inclusion criteria included fluency in English language and comprehension, and those with mild to moderate visual or hearing impairments were also encouraged to take part. Thirty participants (96\%) had corrected vision and four participants had corrected hearing (12\%). One participant (aged 47) had significant visual impairments and was included despite not being an older adult; and was able to complete the trial. Three participants aged between 50 and 59 years were also included on the basis that the first 'older adult' future users of Level 5 CAVs (e.g., within 15-20-years) are likely to currently be aged between 50-60 years. Another three participants were taking antidepressant medication. Despite these factors, all three participants were highly functional and able to complete the trial. Exclusion criteria were: the presence of any severe health conditions (e.g., epilepsy, neurological impairments, heart surgery) that might be aggravated by experimental conditions and manipulations. Participation was voluntary and each participant received a $£ 20$ voucher as a reimbursement for transportation and associated costs.

\subsection{Materials}

\section{HMI}

The design was informed by a literature review [56] and public engagement workshops conducted as part of the Flourish project, and subsequent synthesis of best practices for the design of CAV HMI for older adults from the literature (e.g. reduce clutter, use large icons, use icons that are highly intuitive). The findings resulted in an HMI with several features (e.g. map, date and time, vehicle status, fuel, arrival time, speed, stop and navigation map) that was tested in several simulator-based CAV journeys. As per key findings of our user engagement research, we focus here on a component of the HMI with three main categories of functions: My Profile, Information and Entertainment. Another aspect of the HMI, Future Journey Planning (see Fig. 1) was evaluated after the participants had been in the simulated CAV journeys (post journey), in preparation for the next iteration(s) of the HMI in the next phase of the planned trials. 


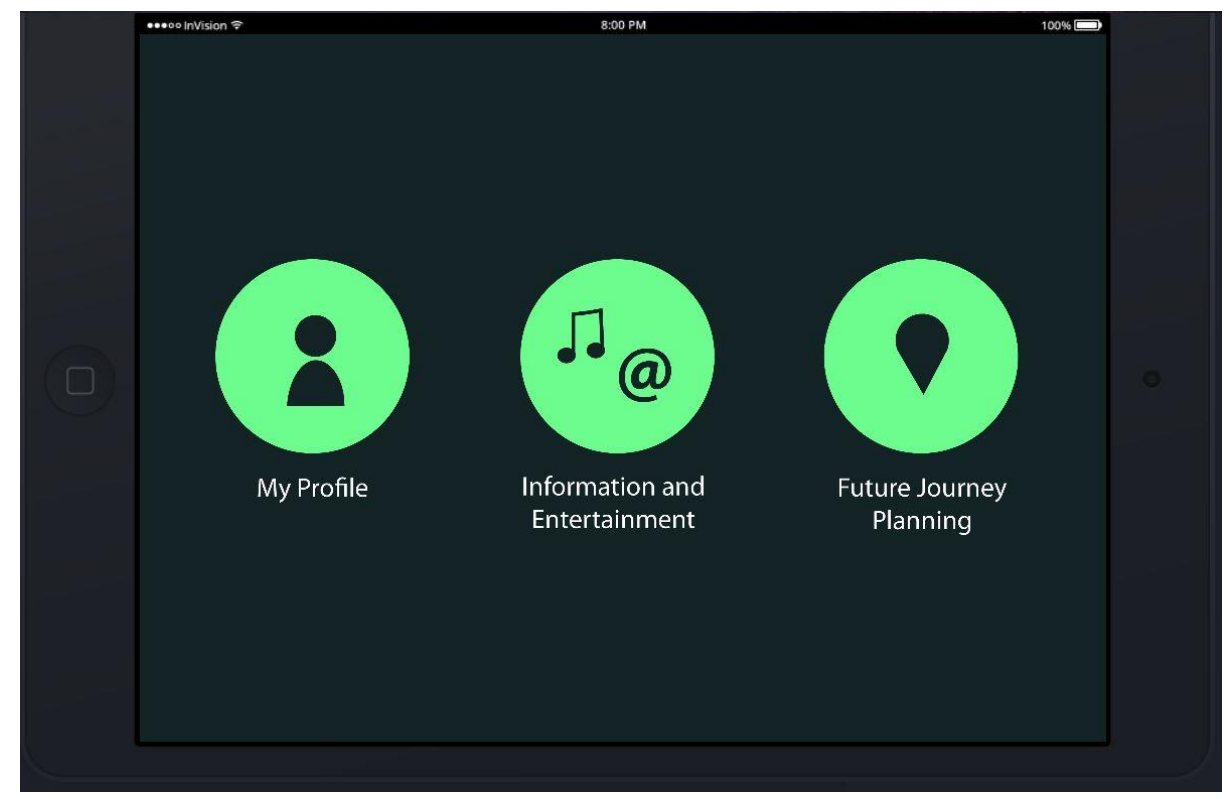

Fig. 1. Overview of the post-journey feature evaluation HMI with three main functions

Each had a drill-down facility to explore related functions. My Profile contained: About $\mathrm{Me}$ (e.g. home address, contacts, health profile) and Preferences (e.g. contrast, brightness, text size) (see. Fig 2).

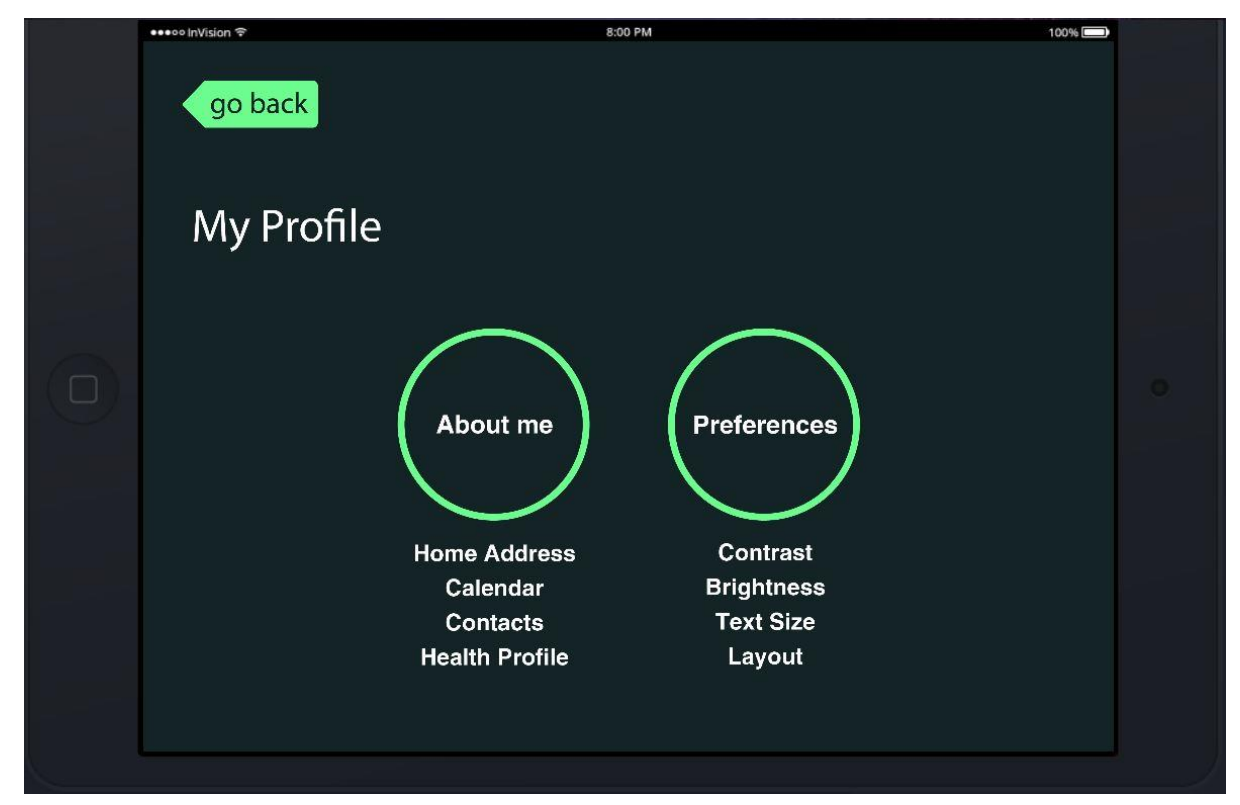

Fig. 2. Drill-down features within the My Profile function 
Information and Entertainment had Television, Internet Search, News, Weather, and Music (see Fig. 3).

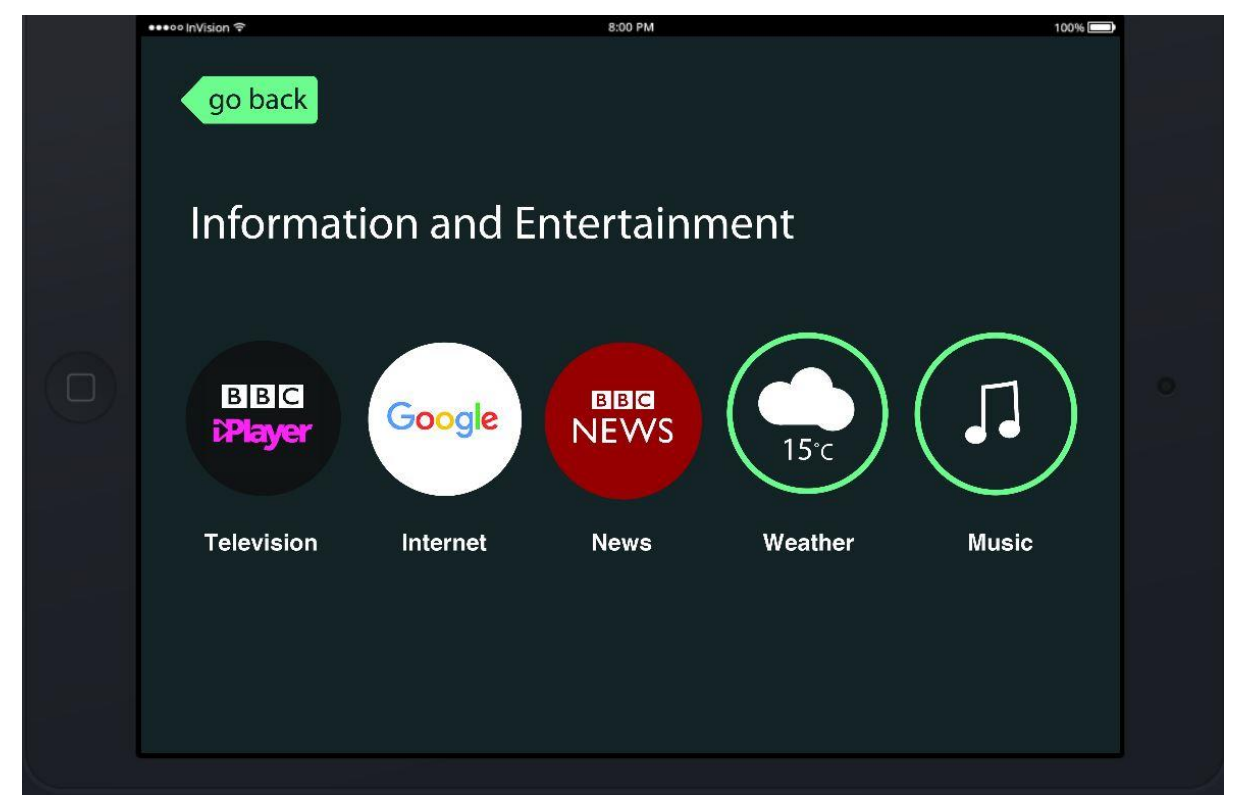

Fig. 3. Drill-down features within the Information and Entertainment function

Future Journey Planning contained Create Future Journey, Planned Journeys, and, Edit Journeys (see Fig. 4). 


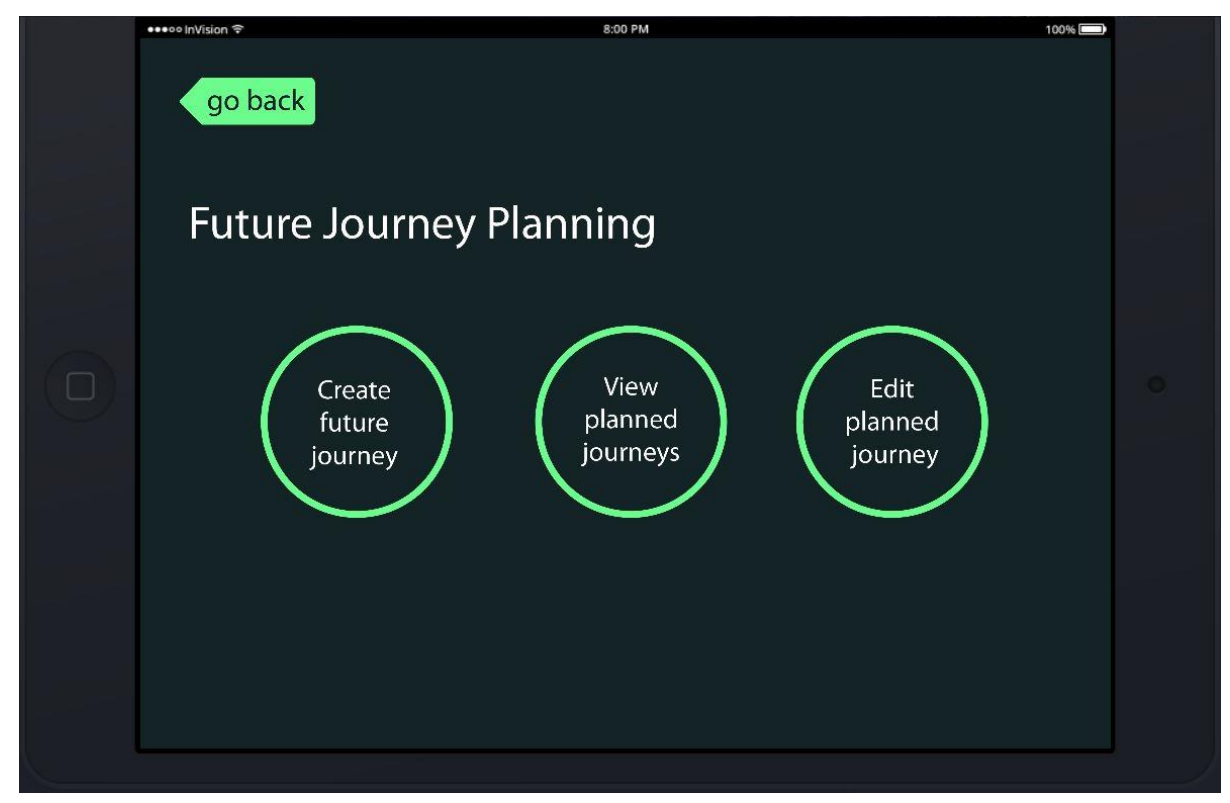

Fig. 4. Drill-down features within the Future Journey Planning function

The post-journey feature evaluation HMI ran on a 12.9 inch iPad Pro with ED backlit display and iPS technology; retina display. It has 2732 x 2048 resolution at 264 pixels per inch, and fingerprint resistant coating.

\subsection{Measures}

Several scales and measures were used. These included:

Self-screening questionnaire. This was used to screen participants for major healthrelated conditions (e.g. stroke, epilepsy).

Demographic questionnaire. This was used to gather data on: age, gender, qualifications, marital and occupational status, and medication taken in the day of testing.

Cognitive functioning. This was assessed using: Ospan [57], Trail Making A \& B Tests [58], and Corsi Blocks Test [59].

A computerized measure of Ospan [57] using Pebl (Version 2.0 Beta 4, [60]) was used to assess working memory capacity task and multi-tasking abilities. The Opsan task consisted of two alternate sub-tasks: 1) remember a serious of two to five letters, and 2) solve math problems (distractor task). The trials were quasi-randomized so that the set size of the next letters and math problems could not be anticipated by the participants. Performance on the letter tasks was calculated by giving equal points to the set size, but only if all the letters from that set were recalled correctly in serial order. This gave an absolute span score. Math accuracy was tracked and participants received feedback during the task. The feedback was included to maintain performance accuracy $\geq 85 \%$ and to keep participants engaged with the task. Two outcomes were used: 1) total 
correct letters recalled and 2) absolute span score. Higher scores reflect better performance.

Trail Making Test Form A \& B (TMT A \& B, [58]). This ran on a LearnPad Android device with Pen Six Screener (PenScreenSix Cognitive Testing Software v2.0 for Android, 2014) and was used as an executive function measure. It has 2 forms: A and B. For A, the participant has to connect, as quickly as possible, 25 encircled numbers in ascending order. For B, the participant tries to connect, as quickly as possible, numbers and letters in ascending order. The outcomes are: (1) time to complete the task, (2) time to complete the task minus the first 2 responses, and (3) number of incorrect responses. Lower scores represent better performance.

Computerized version of Corsi Blocks Test [59]. This was used to measure spatial working memory span. For the task, the participant must tap sequences of blocks arranged irregularly on a board. For each task/trial, blocks on the screen light up one by one in a random order, and the subject must reproduce the order they are lit in. The task includes 12 trials that start with 2 letters and continue up to 7 letters (e.g. 2 trials with 2 letters, 2 trials with 3 letters, 2 trials with 4 letters, etc.). The following outcomes were used: 1) block span, 2) total correct trials, and 3) the product.

Personality. This was assessed using Zuckerman-Kuhlman Personality Questionnaire (ZKPQ-50-CC, shortened form, [45]). The original questionnaire developed by Zuckerman et al. [43]) is based on the Alternative Five Factor Model [43] and measures five personality traits that arguably best describe human behavior: Impulsive Sensation Seeking (e.g. lack of planning, tendency to act quickly on impulse, risk taking, novelty seeking), Aggression Hostility (e.g. antisocial behavior, vengefulness, quick temper), Sociability (e.g. having many friends, enjoying large parties, intolerance for social isolation), Activity (e.g. need for general activity, impatience, preferences for challenging and hard work), and Neuroticism Anxiety (e.g. emotional upset, worry, tension, obsessive indecision). The ZKPQ-50-CC has 50 true-false items, and the total score for each trait is computed by giving a 0 (for a No answers) or 1 (for Yes answers), noting that the questionnaire has reversed items. Original scoring procedure can be found in the original paper $[43,45]$. Higher scores reflect increased personality traits (e.g. someone scoring high on sociability is more sociable than someone scoring low in this trait).

Trust in technology. This was assessed using an adapted version of General Trust in Technology Scale [61]. It contains 7 questions that measure people's trust in technology (e.g. I believe that most technologies are effective at what they are designed to do; I think most technologies enable me to do what I need to do). Responses are recorded using a Likert scale with 1-7 gradations [61]. Higher scores represent increased trust in technology. The scale has reversed items. Higher scores represent increased trust in technology.

Attitudes Towards Computers Questionnaire (ATCQ, [62]). This was used to measure attitudes towards computers and how people relate to computers and whether they are willing to use them for personal or professional reasons. It contains 32 items (e.g. I feel comfortable with computers; Computers are making the jobs done by humans less important) with response options ranging from 5-point Likert scale format ranging from 1 to 5. Lowers scores reflect more negative attitudes towards computers. 
Participants' HMI preferences were measured on a 0 to 10 Likert scale developed by the authors to gain ratings on how useful each function is to them for a Level 4-5 CAV journey. For example, and focussing on a feature of the 'My Profile' function: 'would you find it useful to have an About me function? Please score how you feel on a scale of 0 (completely useless) to 10 (completely useful).' At the end of the survey and after rating the usefulness of each feature, participants were asked to rank the 'My Profile', 'Information and Entertainment' and 'Future Journey Planning' functions based on the likelihood of using them in general. The question was phrased as follow: 'Please tell us how likely you are to use the My Profile, Information and Entertainment, Future Journey Planning functions. Please rank them (1st, 2nd, 3rd)'.

\subsection{Procedure}

The study was approved by the University of the West of England, Bristol, Health and Social Studies (HASS) Research Ethics committee (REF: HAS.16.10.026) with a linked Risk Assessment. Written consent from the participants was obtained prior to the study and after they had received an information sheet. Additionally, each participant had attended an induction workshop (In-Vehicle Participant Workshop - Flourish Deliverable 3.4.2 D3 - In Vehicle Participant Workshop Findings)which was held between 4-8 weeks before the current study depending on when each participant was tested in the main study. The purpose of the workshop was to inform participants about the aims of the project (high level, no predictions), timescale and to gather information relating to e.g., expectancies about autonomous vehicles (e.g., design, journey types, likelihood of using, HMI design features that they would like). It also served as part of an iterative process to design the HMI (noting that the project runs from June 2016May 2019), as well as to keep participants expectations about CAVs to a similar level. (Without this - they could potentially be unequally biased through different personal beliefs and e.g., differing media views of the topic.)

The current HMI evaluation study was part of a larger study within the Flourish project - with an initial component involving participants experiencing journeys within a Level 4-5 simulator and with a basic HMI (not evaluation version) containing e.g., a dynamic map, vehicle status features (such as battery charge remaining, temperature), and a stop button. The screening demographic questionnaire was completed at the beginning of the study. For the evaluation of the HMI component discussed within the current paper, participants sat at a desk with the iPad as described above, and rated preferences on paper after being driven for 4 journeys in the Level 4-5 autonomous simulator. No time constraints were applied. After this, the cognitive tests were completed and not before as they could have induced fatigue. Personality, general trust in technology and attitudes towards computers measures were completed at home, prior to taking part in the main components of the study to avoid responses being affected by experiences during the main study. 


\section{Results and Discussion}

Our first objective was to investigate older adults' preferences of CAV HMI functions. (Note rated out of 10 with 10 being completely useful.) Participants considered the most useful HMI functions to be: Preferences $(M=8.96, S D=1.88)$, Internet search $(M=$ $8.90, S D=1.95)$ and Edit Planned Journeys $(M=8.77, S D=2.14)$. The least useful HMI functions were About me $(M=6.83, S D=3.3)$, News $(M=7.96, S D=2.76)$ and Television $(M=8.36, S D=2.14)$. It is however noted that even though the latter had the lowest usefulness ratings, they were still mostly high $(\geq 6.83 / 10)$ considering a score of 0 translated to completely useless.

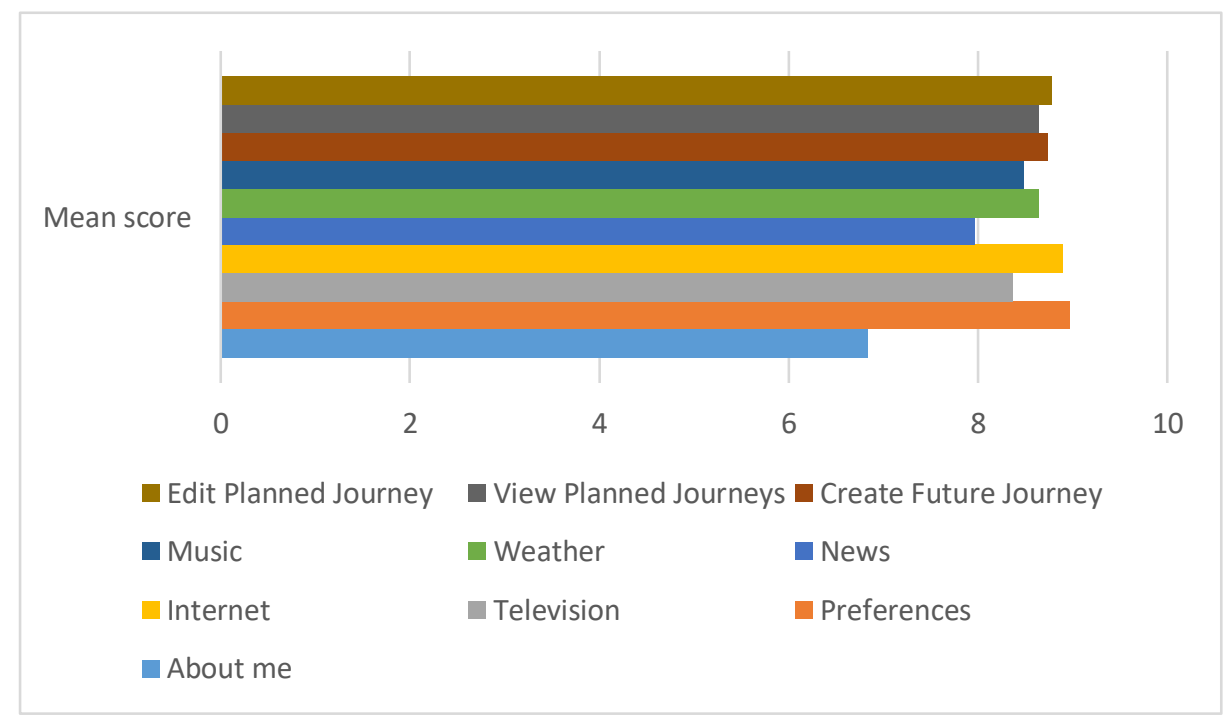

Fig. 5. Preferences for all HMI functions

As for the ranking of the three main functions based on the likelihood of using them within a CAV, the most important function is Future Journey Planning, followed by Information and Entertainment, while My Profile was ranked least important.

Age is not associated with any HMI function preference, and there are no significant differences between younger old participants ( $<70$ years old) and older old $(\geq 70$ years old) adults in terms of their preference for HMI functions, except the Internet search function, which was rated (using an independent-samples t-test) as more likely to be used by the older old participants, $t(29)=-2.00, p<.05$.

Overall, cognitive performance is not significantly associated with preferences for HMI functions.

Executive functioning measured with TMT A is not associated with any HMI functions $(p>.05)$, while TMT B outcomes time to complete task is negatively associated with: Television, Pearson's $r(29)=-.41, p<.05$ and News, Pearson's $r(29)=-.39, p<$ .05 . TMT B time to complete task minus the first 2 responses also correlates negatively with Television, Pearson's $r(29)=-.50, p<.01$ and News, Pearson's $r(29)=-.52, p<$ 
.01. Working memory capacity task and multi-tasking abilities (measured with Ospan) is negatively associated with the Weather Search function, Pearson's $r(29)=.41, p<$ .05 , for Ospan total correct letters recalled, Pearson's $r(29)=.43, p<.05$ for absolute span score. Spatial working memory span measured with the Corsi task is only negatively associated with About Me, for block span outcome, Pearson's $r(29)=-.39, p<$ .05 , and the Corsi product, Pearson's $r(29)=-.40, p<.05$. The correlation between about me and Corsi total correct trials is non-significant, $(p>.05)$.

Personality traits correlate with few preferences for HMI functions. Neuroticism Anxiety seems to be the personality trait that relates to some functions: Television, Pearson's $r=-.49, p<.01$, News, Pearson's $r=-.51, p<.01$, and Weather Search Pearson's $(r=-.45, p<.05)$. Activity correlates positively with Weather search, Pearson's $r=.39, p<.05$. Aggressive Hostility correlates negatively with Television, Pearson's $r=-.42, p<.05$, Sociability correlates with View Planned Journey function, Pearson's $r=-.46, p<.05$. Impulsive Sensation Seeking does not correlate with any function (all $p$ s are not significant). Contrary to our expectations attitudes towards computers and trust in technology do not associate with participants' HMI preferences $(p>.05)$.

The current study aimed to investigate older adults CAV HMI preferences for functions that could be incorporated into an in-vehicle CAV HMI being developed as part of the Innovate UK funded Flourish project. The current study was the first in a series of simulator and road based Level 4-5 CAV trials. We proposed and designed 10 functions that could be integrated in a fully autonomous CAV HMI, including Information and Entertainment (with e.g., Television, Internet, News, Weather, and Music as subfunctions). Noting that some of these (e.g., Television, Internet) would only be feasible in highly automated vehicles where the user does not have to control the driving aspects of the vehicle. In this case, we predicted that users would be less likely to want to access system status and journey related information and focus more on e.g., infotainment functions. Our results show that the sample consisting predominantly of older adults highly likely benefit from an Information and Entertainment function. Their number one choice when it comes to functions that they would use during an CAV journey, given the options provided, is a Future Journey Planner. The My Profile function was rated as the least important. Participants rated Preferences as the most important function out of all 10 proposed functions. This finding is in line with HMI principles recommendation in the literature that endorse increasing adaptability $[1,35]$. This means that our sample of mostly older participants would prefer an HMI with adaptability capabilities that will enable them to change according to their needs the following: contrast, brightness, text size, and layout (see Fig. 2). Interestingly, our participants also preferred the Internet search function and Weather function. Least important functions are News and About me. It might be the case that for our participants, functions such as: home address, calendar, contacts and health profile are either not important or regarded as too personal (e.g., data privacy concerns) [63] and least likely of all functions to be used in in a future CAV journey (see Fig 5).

Individual differences were also shown to have a role in CAV HMI functions preferences amongst our mainly older adult sample. As an example, cognitive abilities seem to relate to some extent to functions preferences. The higher executive functioning abilities, working memory and multi-tasking abilities, the more likely the user will prefer 
functions such as Television, News, About Me, and, Weather search. This might suggest that older adults (again noting the majority of our sample) that score lower on cognitive abilities might prefer to have simpler CAV HMIs with very few features and functions. Though this a tentative conclusion based on an evaluation component of only one study and this area needs further testing.

Some personality traits were shown to have a correlation with CAV HMI preferences, although in general the findings were not consistent and do not suggest clear relationships overall. Interestingly those scoring high on Neuroticism Anxiety are less likely to prefer functions such as: Television, News and Weather search, and those high on Aggressive Hostility are also less likely to prefer the Television function. More active participants were more likely to prefer the Weather Search function, and more sociable participants were more likely to prefer the View Planned Journey function. Contrary to expectation, Impulsive Sensation Seeking does not relate to any HMI function preference. Similarly, neither trust in technology, nor attitudes towards computers correlate with any HMI function that were tested as part of this evaluation study. It might be the case that such variables do not shape or relate to a particular HMI function preference because older people (generally), despite their level of trust in technology and attitudes towards computers, prefer to interact with HMI functions. However, our results should not be interpreted out of the context, as we did not test these functions during an actual CAV journey (i.e., this was an evaluation component of a larger study and the HMI features rated were not a feature within the component that involved experiencing simulated CAV journeys). During an autonomously driven journey, participants might have other preferences. Our study is a starting point for future studies that aim to develop CAV HMIs for use amongst older adults, including those currently underway and planned as part of our Flourish project.

\section{Implications for HMI Design}

Our work is highly relevant for the design of CAV HMIs for older adults, noting the conclusions drawn are based on an evaluation component of one study that assessed participants' HMI preferences after exposure in a CAV simulator. First it offers an insight into older adult's preferences for functions that could be available within highly and fully autonomous (Levels 4-5) CAVs. The findings indicate that our sample of participants also prefer the idea of adaptive HMIs. Features such as adaptable contrast, brightness, text size, and layout are welcomed and likely to be used. This might help those with various age-related sensory difficulties. (Noting that most of our participants had corrected vision.) Functions that keep participants engaged in other activities during a L5 CAV journey, such as Information and Entertainment (e.g., Internet, Weather, Television, Music), were also evaluated positively. Thus, such features should be investigated and developed further and tested during actual Level 4-5 CAV journeys. We found the most important general function is a journey planner. This is a rather intuitive finding given that Level 4-5 CAVs are being designed in a way that the user no longer 
needs to drive between destinations and therefore forward planning (i.e., journey setting) is important, much like having saved numbers on a telephone agenda as we no longer need to commit them to memory.

From an individual differences perspective, some personal characteristics like personality traits seem to have a reduced role in the CAV HMI preferences participants have, but this conclusion is based on an evaluation component of only one study and outside a CAV simulator experience. Therefore, future studies might focus on this area of research, by conducting HMI evaluations during actual CAV simulated journeys, or even CAV journeys. One key finding is that scoring lower on cognitive tests makes it harder to engage with HMI functions, as a good cognitive performance is associated with increased HMI functions preference. Personality as well has limited association with HMI function preferences tested as part of the current study, as only some personality traits relate with a few functions. For instance, people that have a tendency to worry in excess or to be anxious might be less likely to prefer HMI functions as Television, News and Weather search, and those very active might prefer a Weather search function. For risk takers and impulsive individuals, our results didn't show propensity for any particular HMI preferences. Finally, it is interesting that attitudes towards computers and trust in technology did not relate to any HMI function preferences either, even though studies suggest that technology related measures predict the use of technology [50,51,64-66]. A possible explanation could be the design of the current study, as participants had to rate their HMI preferences during an evaluation phase and not during simulated CAV journeys. It might be the case that during a Level 4-5 CAV journey, participants might prioritize some functions compared to their current ratings in a passive evaluation study.

Overall, our findings speak to current and future designs of Level 4-5 CAV HMIs for older adults by making them more adaptable and easy to use. User friendly functions that are intuitive and easy understand are likely to increase the likelihood of a predominantly older adult sample using them. This might also help reduce possible anxieties related to the functions and their functionality given that $\mathrm{AVs} / \mathrm{CAVs}$ are still a relative new and emerging technology. Users need to build up experience and trust with such features and functions (and others). Also, future evaluation studies (including those with pre-, during-, and post- $\mathrm{CAV}$ journey exposure and usage) are needed to speak to their longer-term inclusion and/or development for use within HMIs integrated into road-based CAVs. Our extensive battery of scales and measures used in this study show that some of these could be used to preempt user preferences and pre-customize the interface as part of a richer personalized CAV experience. This will be explored further as part of future work.

\section{Acknowledgments.}

The reported research forms part of an Innovate UK research project -FLOURISH: Empowerment through Trusted Secure Mobility (2016-2019). See http://flourishmobility.com/ 
We are thankful to our collaborators at Designability UK (Jessica Ridgers, Keir Haines, Hazel Boyd) for designing the HMI based upon best HF principles and participant evaluation at a workshop proceeding the current study, and, collaborators at Transport Systems Catapult UK (Alan Nettleton, Duncan Napier, and Sophie N'Guende) for programming the HMI used within the simulator component of the larger study.

\section{References}

1. Morgan, P.L., Voinescu, A., Williams, C., Caleb-Solly, P., Alford, C., Shergold, I., Pipe, A.: An Emerging Framework to Inform Effective Design of Human-Machine Interfaces for Older Adults Using Connected Autonomous Vehicles. In: International Conference on Applied Human Factors and Ergonomics 2017, pp. 325-334. Springer, Cham

2. Morgan, P., Alford, C., Parkhurst, G.: Handover issues in autonomous driving: A literature review. Project Report. (2016).

3. Webber, S.C., Porter, M.M., Menec, V.H.: Mobility in older adults: a com-prehensive framework. The Gerontologist 50(4), 443-450 (2010).

4. Chihuri, S., Mielenz, T.J., DiMaggio, C.J., Betz, M.E., DiGuiseppi, C., Jones, V.C., Li, G.: Driving cessation and health outcomes in older adults. Journal of the American Geriatrics Society 64(2), 332-334 (2016).

5. Davis, J.C., Bryan, S., Li, L.C., Best, J.R., Hsu, C.L., Gomez, C., Liu-Ambrose, T.: Mobility and cognition are associated with wellbeing and health related quality of life among older adults: a cross-sectional analysis of the Vancouver Falls Prevention Cohort. BMC Geriatrics 15(75), 17 (2015).

6. Fagnant, D.J., Kockelman, K.: Preparing a nation for autonomous vehicles: opportunities, barriers and policy recommendations. Transportation Research Part A: Policy and Practice 77, 167-181 (2015).

7. Stanton, N.A., Salmon, P.M.: Human error taxonomies applied to driving: A generic driver error taxonomy and its implications for intelligent transport systems. Safety Science 47(2), $227-$ 237 (2009).

8. Dotzauer, M., De Waard, D., Caljouw, S.R., Pöhler, G., Brouwer, W.H.: Behavioral adaptation of young and older drivers to an intersection crossing advisory system. Accident Analysis \& Prevention 74, 24-32 (2015).

9. Siren, A., Haustein, S.: Driving licences and medical screening in old age: re-view of literature and European licensing policies. Journal of Transport \& Health 2(1), 68-78 (2015).

10. Pachana, N.A., Jetten, J., Gustafsson, L., Liddle, J.: To be or not to be (an older driver): social identity theory and driving cessation in later life. Ageing \& Society 37(8), 1597-1608 (2017).

11. Marottoli, R.A., Leon, C.F.M., Glass, T.A., Williams, C.S., Cooney, L.M., Berkman, L.F., Tinetti, M.E.: Driving cessation and increased depressive symptoms: Prospective evidence from the New Haven EPESE. Journal of the American Geriatrics Society 45(2), 202-206 (1997).

12. Choi, M., Lohman, M.C., Mezuk, B.: Trajectories of cognitive decline by driv-ing mobility: evidence from the Health and Retirement Study. International Journal of Geriatric Psychiatry 29(5), 447-453 (2014). 
13. International, S.: U.S. Department of transportation's new policy on automated vehicles adopts SAE International's levels of automation for defining driving automation in on-road motor vehicles. In. (2016)

14. Riener, A., Boll, S., Kun, A.L.: Automotive user interfaces in the age of automation (Dagstuhl Seminar 16262). In: Dagstuhl Reports vol. 6. (2016)

15. Merat, N., Lee, J.D.: Preface to the special section on human factors and automation in vehicles: Designing highly automated vehicles with the driver in mind. Human Factors 54(5), 681-686 (2012).

16. Merat, N., Jamson, A.H., Lai, F.C., Daly, M., Carsten, O.M.: Transition to manual: Driver behaviour when resuming control from a highly automated vehicle. Transportation Research Part F: Traffic Psychology and Behaviour 27, 274-282 (2014).

17. Eriksson, A., Banks, V.A., Stanton, N.A.: Transition to manual: Comparing simulator with on-road control transitions. Accident Analysis \& Prevention 102, 227-234 (2017).

18. Stanton, N.A., Young, M.S.: A proposed psychological model of driving automation. Theoretical Issues in Ergonomics Science 1(4), 315-331 (2000).

19. De Winter, J.C., Happee, R., Martens, M.H., Stanton, N.A.: Effects of adaptive cruise control and highly automated driving on workload and situation awareness: A review of the empirical evidence. Transportation Research Part F: Traffic Psychology and Behaviour 27, 196-217 (2014). 20. Onnasch, L., Wickens, C.D., Li, H., Manzey, D.: Human performance consequences of stages and levels of automation: An integrated meta-analysis. Human Factors 56(3), 476-488 (2014).

21. Deary, I.J., Corley, J., Gow, A.J., Harris, S.E., Houlihan, L.M., Marioni, R.E., Starr, J.M.: Age-associated cognitive decline. British Medical Bulletin 92(1), 135-152 (2009).

22. Taylor, W.D., Raji, C., Wang, L., \& Lavretsky, H. (2016). Can We Change the Inevita-ble? Ameliorating Brain Aging and Cognitive Decline. The American Journal of Geriat-ric Psychiatry, 24(3), S7.: Can We Change the Inevitable? Ameliorating Brain Aging and Cognitive Decline. The American Journal of Geriatric Psychiatry 24(3), S7 (2016).

23. Philip, P., Taillard, J., Sagaspe, P., Valtat, C., Sanchez-Ortuno, M., Moore, N., Bioulac, B.: Age, performance and sleep deprivation. Journal of Sleep Research 13(2), 105-110 (2004).

24. McGwin Jr, G., Sims, R.V., Pulley, L., Roseman, J.M.: Relations among chronic medical conditions, medications, and automobile crashes in the elderly: a population-based case-control study. American Journal of Epidemiology 152(5), 424-431 (2000).

25. Hartshorne, J.K., Germine, L.T.: When does cognitive functioning peak? The asynchronous rise and fall of different cognitive abilities across the life span. Psychological science 26(4), 433443 (2015).

26. Bopp, K.L., Verhaeghen, P.: Aging and verbal memory span: A meta-analysis. The Journals of Gerontology Series B: Psychological Sciences and Social Sciences 60(5), P223-P233 (2005). 27. Verhaeghen, P., Cerella, J.: Aging, executive control, and attention: a review of metaanalyses. Neuroscience \& Biobehavioral Reviews 26(7), 849-857 (2002).

28. Lee, H.K., Scudds, R.J.: Comparison of balance in older people with and without visual impairment. Age and Ageing 32(6), 643-649 (2003).

29. Prince, M.J., Wu, F., Guo, Y., Robledo, L.M.G., O'Donnell, M., Sullivan, R., Yusuf, S.: The burden of disease in older people and implications for health policy and practice. The Lancet 385(9967), 549-562 (2015). 
30. Quaranta, N., Coppola, F., Casulli, M., Barulli, O., Lanza, F., Tortelli, R., Solfrizzi, V.: The prevalence of peripheral and central hearing impairment and its relation to cognition in older adults. Audiology and Neurotology 19(Suppl. 1), 10-14 (2014).

31. Seubert, J., Laukka, E.J., Rizzuto, D., Hummel, T., Fratiglioni, L., Bäckman, L., Larsson, M.: Prevalence and correlates of olfactory dysfunction in old age: A population-based study. Journals of Gerontology Series A: Biomedical Sciences and Medical Sciences glx054 (2017).

32. Varma, V.R., Hausdorff, J.M., Studenski, S.A., Rosano, C., Camicioli, R., Alexander, N.B., Carlson, M.C.: Aging, the central nervous system, and mobility in older adults: interventions. Journals of Gerontology Series A: Biomedical Sciences and Medical Sciences 71(11), 1451-1458 (2016).

33. Bruder, C., Blessing, L., Wandke, H.: Adaptive training interfaces for less-experienced, elderly users of electronic devices. Behaviour \& Information Technology 33(1), 4-15 (2014).

34. Young, K.L., Koppel, S., Charlton, J.L.: Toward best practice in human machine interface design for older drivers: a review of current design guidelines. Accident Analysis \& Prevention 106, 460-467 (2017).

35. Fisk, A.D., Czaja, S.J., Rogers, W.A., Charness, N., Sharit, J.: Designing for older adults: Principles and creative human factors approaches. CRC press, Florida, FL (2009)

36. Williams, D., Alam, M.A.U., Ahamed, S.I., Chu, W.: Considerations in designing humancomputer interfaces for elderly people. In: Gotlieb, A., Chen, Z. (eds.) 13th International Conference on Quality Software, China, July 2013, pp. 372-377. IEEE

37. Eisses, S.: ITS Action Plan. RappTrans. . European commission D4 Final Report (2011).

38. Emmerson, C., Guo, W., Blythe, P., Namdeo, A., Edwards, S.: Fork in the road: In-vehicle navigation systems and older drivers. Transportation Research Part F: Traffic Psychology and Behaviour 21, 173-180 (2013).

39. Molnar, L.J., Eby, D.W.: Implications of advanced vehicle technologies for older drivers. Accident Analysis and Prevention 106, 457-459 (2017).

40. Duan, Y.A., Song, H.T., Sun, Y.M.: The user-oriented design and practice of HMI. Applied Mechanics and Materials, Trans Tech Publications 58, 1819-1823 (2011).

41. Gentner, A., Bouchard, C., Favart, C.: Investigating user experience as a composi-tion of components and influencing factors. In: 5th International Congress of In-ternational Association of Societies of Design Research, Consilence and Innovation in Design - Proceedings and Program, Tokyo 2013, pp. 0319-3330

42. Engström, J., Arfwidsson, J., Amditis, A., Andreone, L., Bengler, K., Cacciabue, P.C., Nathan, F.: Towards the automotive HMI of the future: mid-term results of the AIDE project. In: Valldorf, J., Gessner, W. (eds.) Advanced Microsystems for Automotive Applications 2006. Springer

43. Zuckerman, M.: Sensation Seeking (psychology revivals): Beyond the optimal level of arousal. Psychology Press, London, UK (2014)

44. Wang, Z., Vang, M., Lookadoo, K., Tchernev, J.M., Cooper, C.: Engaging high-sensation seekers: The dynamic interplay of sensation seeking, message visual-auditory complexity and arousing content. Journal of Communication 65(1), 101-124 (2014).

45. Aluja, A., García, Ó., García, L.F.: A comparative study of Zuckerman's three structural models for personality through the NEO-PI-R, ZKPQ-III-R, EPQ-RS and Goldberg's 50-bipolar adjectives. Personality and Individual Difference 33(5), 713-725 (2002). 
46. Ekman, F., Johansson, M., Sochor, J.: Creating appropriate trust for autono-mous vehicle systems: A framework for HMI Design. Paper presented at the Transportation Research Board 95th Annual Meeting (No. 16-3268), Washington, D.C.,

47. Mirnig, A.G., Wintersberger, P., Sutter, C., Ziegler, J.: A framework for analyzing and calibrating trust in automated vehicles. In: Proceedings of the 8th In-ternational Conference on Automotive User Interfaces and Interactive Vehicular Applications Adjunct Ann Arbor, MI, October 2016, pp. 33-38. ACM

48. Hoff, K.A., Bashir, M.: Trust in automation: Integrating empirical evidence on factors that influence trust. Human Factors 57(3), 407-434 (2015).

49. Forster, Y., Naujoks, F., Neukum, A.: Increasing anthropomorphism and trust in automated driving functions by adding speech output. In: ntelligent Vehicles Symposium (IV), Los Angeles, CA 2017, pp. 365-372. IEEE

50. Agarwal, R., Karahanna, E.: Time flies when you're having fun: Cognitive absorption and beliefs about information technology usage. MIS quarterly 24(4), 665-694 (2000). doi: $10.2307 / 3250951$

51. Czaja, S.J., Charness, N., Fisk, A.D., Hertzog, C., Nair, S.N., Rogers, W.A., Sharit, J.: Factors predicting the use of technology: findings from the Center for Re-search and Education on Aging and Technology Enhancement (CREATE). Psychology and Aging 21(2), 333-352 (2006).

52. Aula, A.: Learning to use computers at a later age. HCI and the Older Population 3, 1-4 (2004).

53. Tsai, H.Y.S., Shillair, R., Cotten, S.R., Winstead, V., Yost, E.: Getting grand-ma online: are tablets the answer for increasing digital inclusion for older adults in the US? Educational Gerontology 4(10), 695-709 (2015).

54. González, A., Ramírez, M.P., Viadel, V.: ICT learning by older adults and their attitudes toward computer use. Current Gerontology and Geriatrics Research 2015, 1-7 (2015).

55. Cohen, J.: Statistical Power Analysis for the Behavioral Sciences, 2 ed. La Wrence Erlbaum Associates, Publishers, Hillsdale, New Jersey (1988)

56. Morgan, P., Caleb-Solly, P., Voinescu, A., Williams, C.: Literature Review: Human-Machine Interface. Project report. In. UWE Bristol, Bristol, (2016)

57. Turner, M.L., Engle, R.W.: Is working memory capacity task dependent? Journal of Memory \& Language 28, 127-154 (1989).

58. Reitan, R.M.: Validity of the Trail Making Test as an indicator of organic brain damage. Perceptual and Motor Skills 8(3), 271-276 (1958). doi:10.2466/pms.1958.8.3.271

59. Corsi, P.: Memory and the medial temporal region of the brain. McGill University, (1972) 60. Mueller, S.T., Piper, B.J.: The Psychology Experiment Building Language (PEBL) and PEBL Test Battery. Journal of neuroscience methods 222, 250-259 (2014).

61. Mcknight, D.H., Carter, M., Thatcher, J. B., \& Clay, P. F. (2011). Trust in a specific technology: An investigation of its components and measures. ACM Transactions on Management Information Systems (TMIS), 2(2), 12.: Trust in a specific technology: An investigation of its components and measures. ACM Transactions on Management Information Systems (TMIS) 2(2), 1-24 (2011).

62. Jay, G.M., Willis, S.L.: Influence of direct computer experience on older adults' attitudes toward computers. Journal of Gerontology 47(4), P250-P257 (1992). 
63. Chakraborty, R., Vishik, C., Rao, H.R.: Privacy preserving actions of older adults on social media: Exploring the behavior of opting out of information sharing. Decision Support Systems 55(4), 948-956 (2013).

64. Kennedy, R.S., Lane, N.E., Berbaum, K.S., Lilienthal, M.G.: Simulator sickness questionnaire: An enhanced method for quantifying simulator sickness. The international journal of aviation psychology 3(3), 203-220 (1993). doi:10.1207/s15327108ijap0303_3

65. Laguna, K., Babcock, R.L.: Computer anxiety in young and older adults: Implications for human-computer interactions in older populations. Computers in Human Behavior 13(3), 317326 (1997).

66. Witmer, B.G., \& Singer, M. J.: Measuring presence in virtual environments: A presence questionnaire. Presence: Teleoperators and virtual environments 7(3), 225-240 (1998). 